\title{
Effects of a single aerobic exercise on perfused boundary region and microvascular perfusion: a field study
}

\author{
Alexander Fuchs $^{1}$ (D) $\cdot$ Tobias Neumann $^{1} \cdot$ Hendrik Drinhaus ${ }^{1} \cdot$ Anika Herrmann $^{1} \cdot$ Hans Vink $^{2} \cdot$ Thorsten Annecke $^{1,3}$
}

Received: 2 August 2020 / Accepted: 16 January 2021 / Published online: 3 February 2021

(c) The Author(s) 2021

\begin{abstract}
The endothelium and the glycocalyx play a pivotal role in regulating microvascular function and perfusion in health and critical illness. It is unknown today, whether aerobic exercise immediately affects dimensions of the endothelial surface layer (ESL) in relation to microvascular perfusion as a physiologic adaption to increased nutritional demands. This monocentric observational study was designed to determine real-time ESL and perfusion measurements of the sublingual microcirculation using sidestream dark field imaging performed in 14 healthy subjects before and after completing a $10 \mathrm{~km}$ trial running distance. A novel image acquisition and analysis software automatically analysed the perfused boundary region (PBR), an inverse parameter for red blood cell (RBC) penetration of the ESL, in vessels between 5 and $25 \mu \mathrm{m}$ diameter. Microvascular perfusion was assessed by calculating RBC filling percentage. There was no significant immediate effect of exercise on PBR and RBC filling percentage. Linear regression analysis revealed a distinct association between change of PBR and change of RBC filling percentage (regression coefficient $\beta:-0.026 ; 95 \%$ confidence interval -0.043 to $-0.009 ; \mathrm{p}=0.006$ ). A single aerobic exercise did not induce a change of PBR or RBC filling percentage. The endothelium of the microvasculature facilitates efficient perfusion in vessels reacting with an increased endothelial surface layer.
\end{abstract}

Keywords Endothelial surface layer · Glycocalyx $\cdot$ Perfused boundary region · Capillary density $\cdot$ Intravital microscopy Sidestream darkfield imaging

\section{Abbreviations \\ ESL Endothelial surface layer \\ PBR Perfused boundary region \\ RBC(s) Red blood cell(s)}

\section{Introduction}

Microvascular perfusion is adapted to local needs by finetuned adaptive mechanism. In this context, the endothelial glycocalyx and the endothelium play a pivotal role in

Thorsten Annecke

annecket@kliniken-koeln.de

1 Faculty of Medicine and University Hospital of Cologne, Department of Anaesthesiology and Intensive Care Medicine, University of Cologne, Cologne, Germany

2 Department of Physiology, Maastricht University Medical Center, Maastricht, The Netherlands

3 Department of Anaesthesiology and Intensive Care Medicine, University of Witten/ Herdecke, Kliniken der Stadt Köln gGmbH, Cologne, Germany regulating microvascular function and perfusion. The glycocalyx is composed of proteoglycans and glycosaminoglycans on its luminal site. It is part of the endothelial surface layer (ESL) that contains associated soluble plasma proteins and immobilised plasma. The ESL is involved in many physiological functions such as mechanotransduction, haemostasis, signalling, and blood cell-vessel wall interactions [1, 2]. Given these essential functions, ESL degradation will lead to formation of tissue edema, loss of nutritional blood flow and leucocyte adhesion in states of critical illness such as sepsis, ischaemia-reperfusion-injury and trauma [2]. Experimental models of ischaemia/reperfusion and hypoxia/reoxygenation showed that myocardial hypoxia and ischaemia induce an early ESL degradation [3], with purin signalling and perivascular mast cell-derived heparanase being a possible contributing factor [4]. Increased blood levels of shed ESL components such as syndecan-1, hyaluronan and heparan sulphate can be detected in successful cardiopulmonary resuscitation after cardiac arrest, trauma and sepsis and may serve as an early predictor of adverse clinical outcome [5-7]. Soluble components of the endothelial glycocalyx may act as damage associated molecular patterns (DAMPs), further 
augmenting inflammation and tissue damage. Therefore, eliminating these substances from the circulation is under evaluation as a therapeutic intervention [8].

In order to visualise and calculate ESL dimensions, novel non-invasive techniques are available to study the microcirculation in regions that are easily accessible. Recently, clinical studies using intravital microscopy of the sublingual microvasculature have been conducted to assess the ESL in various medical conditions and critical care states [9-14]. These measurements were performed in patients and controls either in-hospital or in a laboratory setting.

However, rapid changes of ESL dimensions may occur. To this date, it is unknown whether aerobic exercise immediately affects dimensions of the endothelial surface layer and hereby adapts local tissue perfusion. The current study aimed to investigate the microvascular function of healthy adults using the above mentioned non-invasive techniques before and after running a $10 \mathrm{~km}$ trial distance. We hypothesised that aerobic exercise will induce a physiological change of ESL dimensions in order to facilitate efficient perfusion. Furthermore, we tested the feasibility of postexercise on-site measurements to elucidate alternative scenarios for intravital microscopy other than an in-hospital or a laboratory setting.

\section{Methods}

\subsection{Study design, setting and subjects}

This was a monocentric, observational field study to obtain the effect of aerobic exercise on real-time ESL and perfusion measurements of the sublingual microcirculation in 14 healthy adults. Measurements were performed on site before and after running a $10 \mathrm{~km}$ distance trail race consisting of 4 rounds with a total difference in altitude of $72 \mathrm{~m}$ in a public park. The $10 \mathrm{~km}$ route had been measured and predefined beforehand. Transponder timing of every participant had been established. A generator was used to meet the power demand of the videomicroscope and ensure the performance of the connected notebook. Upon enrolment, every participant was asked to fill in a questionnaire about health and long-term medication. Standard vital parameters were obtained before and after the endurance run.

The primary endpoint was the perfused boundary region (PBR) of vessels between 5 and $25 \mu \mathrm{m}$ diameter as an inverse parameter for ESL dimensions. PBR is the depth of lateral erythrocyte penetration into ESL. Its calculation has been described elsewhere [15]. Secondary endpoints were PBR of the sublingual microvasculature graded according to vessel size, red blood cell (RBC) filling percentage as a parameter for microvascular perfusion and change of the mentioned parameters between post-exercise and baseline. Valid and total capillary density as well as the ratio of valid to total capillary density served as an additional marker for microvascular perfusion and capillary recruitment, respectively.

The study was conducted in accordance with the Declaration of Helsinki and its protocol has been approved by the local Ethics Committee of the University of Cologne (AZ 18-162). All participants gave written informed consent prior to any study-related procedures.

\subsection{Assessment of ESL dimensions and microvascular perfusion}

Participants underwent non-invasive imaging of the sublingual microvasculature via intravital microscopy using a handheld sidestream darkfield videomicroscope (Capiscope HVCS Handheld Video Capillaroscopy System, KK Research Technology, Devon, UK). Properties of the endothelial surface layer and quality of microvascular perfusion were analysed using GlycoCheck ${ }^{\mathrm{TM}}$ software (Microvascular Health Solutions, Orem, Utah, USA). During acquisition the software provides feedback regarding stability and focus and adjusts light intensity. This ensures that only adequate images are being recorded for automatic analysis and thus limits inter-observer variability.

Each participant underwent two baseline und two postexercise measurements from which the mean PBR was calculated to provide a single PBR value per subject per measurement. The process of image acquisition and reproducibility of imaging and analysis via intravital microscopy and the above mentioned software have been described elsewhere [12-14].

The perfused boundary region (PBR) is an inverse parameter for RBC penetration of the endothelial surface layer, and hence, its thickness. RBC filling percentage is the percentage of time in which valid vessel segments have RBCs present and is an estimate for microvascular perfusion [15]. RBC filling percentage is determined in individual vascular segments and is defined as the fractional longitudinal RBC content without assessing haemodynamics. The presence of RBCs along the length of a vascular segment is determined by measuring radial intensity profiles at 21 positions in 40 sequential video frames, resulting in 840 potential RBC detections. RBC filling percentage is the number of positive $\mathrm{RBC}$ detections relative to the maximal number of 840 . The change of PBR and RBC filling percentage was calculated by subtracting the baseline from the post-exercise value. The detailed definition of valid and total capillary density is provided elsewhere [16].

\subsection{Statistical analysis}

As this was a pilot study and a field test for the method itself, we did not conduct a sample size estimation. Analysis 
of within-group differences was carried out using Wilcoxon test. Normally distributed variables are presented as mean \pm standard deviation; for non-normally distributed data the median and interquartile range are given. Spearman's correlation was used to test independence between variables. Linear regression analysis was performed to investigate the associations of the change of PBR and change of RBC filling percentage. The significance threshold was set at 0.05 (twotailed). Calculations were performed with SPSS Statistics v25 (IBM Corp., Armonk, NY, USA). Data visualisation was conducted in RStudio 1.2.5019 for R [17] with the package 'gplot2' [18].

\section{Results}

\subsection{Study population}

Participants' clinical and demographic characteristics preand post-exercise are displayed in Table 1 . Fourteen young to middle-aged, normal weight, normotensive adults were included, a third being female. Ten kilometre trial running time ranged from 48 to $59 \mathrm{~min}$. Post-exercise there was an increase of heart rate accompanied by a slight decrease in systolic blood pressure and peripheral oxygen saturation.

\subsection{ESL dimension and perfusion measurements}

The baseline PBR of vessels between 5 and $25 \mu \mathrm{m}$ diameter was 1.86 [1.72-2.08] $\mu \mathrm{m}$. Baseline RBC filling percentage was $75.6[72.1-79.2] \%$. There was no significant immediate effect of aerobic exercise on PBR of 5 to $25 \mu \mathrm{m}$ vessels (1.82 [1.72-2.11] $\mu \mathrm{m}, \mathrm{p}=0.975), \mathrm{RBC}$ filling percentage (74.6 [69.8-80.0]\%, $\mathrm{p}=0.397$ ) (Fig. 1) or differentiated PBR according to vessel size (i.e. 5 to 9,10 to 19 and 20 to $25 \mu \mathrm{m})$ (data not shown).

The baseline valid capillary density was 453 [340-521] n/ $\mathrm{mm}^{2}$ and the total capillary density $731[614-823] \mathrm{n} / \mathrm{mm}^{2}$. There was no significant immediate effect on valid capillary density (457 [350-492] n/mm $\left.{ }^{2}, \mathrm{p}=0.660\right)$ or total capillary density post-exercise $\left(693[616-904] \mathrm{n} / \mathrm{mm}^{2}, \mathrm{p}=0.950\right)$. The baseline ratio of valid to total capillary density was 57 [48-67]\%. There was no significant immediate effect of aerobic exercise on the ratio of valid to total capillary density $(60[49-67] \%, \mathrm{p}=0.975)$.

PBR and RBC filling percentage at baseline, PBR and RBC filling percentage at post-exercise (Fig. 2) as well as change of PBR and change of RBC filling percentage showed a strong negative correlation, respectively (Fig. 3). Linear regression analysis revealed a distinct association between change of PBR and change of RBC filling percentage (regression coefficient $\beta:-0.026 ; 95 \%$ confidence interval: -0.043 to $-0.009 ; \mathrm{p}=0.006$ ).

\section{Discussion}

We were not able to detect an immediate effect on ESL dimensions following aerobic exercise in this study. There was a negative association of PBR and RBC filling percentage at baseline, as has been described in previous studies [9, $15,16]$. This negative association of PBR and RBC filling

Table 1 Participant characteristics and pre- and post-exercise vital parameters

\begin{tabular}{|c|c|c|c|}
\hline & & & $\mathrm{n}=14$ \\
\hline Age (years) & & & $36 \pm 8$ \\
\hline Gender (female) & & & $5(35.7)$ \\
\hline Height (m) & & & $1.82[1.74-1.88]$ \\
\hline Weight (kg) & & & $75 \pm 12$ \\
\hline BMI $\left(\mathrm{kg} / \mathrm{m}^{2}\right)$ & & & $22.9 \pm 3.0$ \\
\hline Men & & & $23.9 \pm 3.2$ \\
\hline \multirow[t]{2}{*}{ Women } & & & $21.2 \pm 1.5$ \\
\hline & Baseline & Post-exercise & $\mathrm{p}$ \\
\hline Heart rate (beats/min) & $70[65-77]$ & 105 [95-118] & 0.001 \\
\hline Systolic blood pressure (mmHg) & $125[120-140]$ & $120[110-130]$ & 0.012 \\
\hline Diastolic blood pressure $(\mathrm{mmHg})$ & $80[70-90]$ & $80[80-90]$ & 0.217 \\
\hline $\mathrm{SpO}_{2}(\%)$ & 98 [98-98] & 97 [95-97] & 0.021 \\
\hline Ten kilometre running time (mm:ss) & $\begin{array}{l}53: 26 \pm 03: 42 \\
51: 38[51: 11-57: 48]\end{array}$ & & \\
\hline
\end{tabular}

$B M I$ body mass index, $\mathrm{SpO}_{2}$ peripheral oxygen saturation

Values are given as mean \pm standard deviation, frequency (percentage) median or median [1. quartile -3 . quartile] 
Fig. 1 a PBR $(\mu \mathrm{m})$ in vessels between 5 and $25 \mu \mathrm{m}$ diameter at baseline and post-exercise as an inverse parameter for $\mathrm{RBC}$ penetration into the ESL. b RBC filling (\%) at baseline and post-exercise. The whiskers extend from Q1 and Q3 to minimum and maximum of the data sets

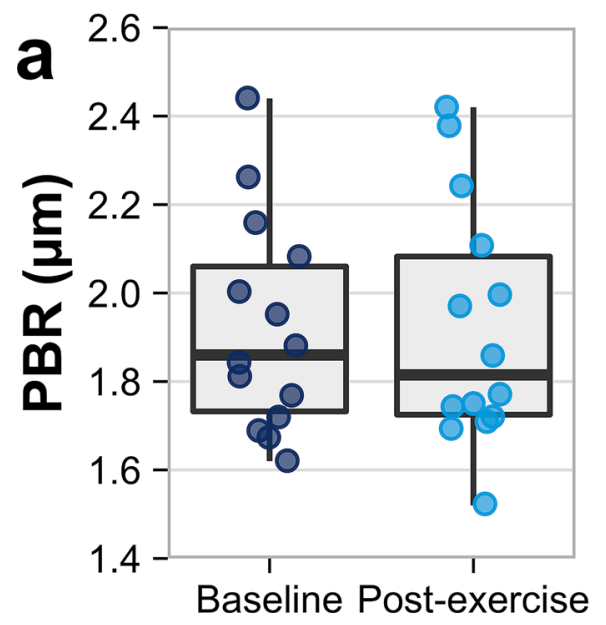

\section{a Baseline}

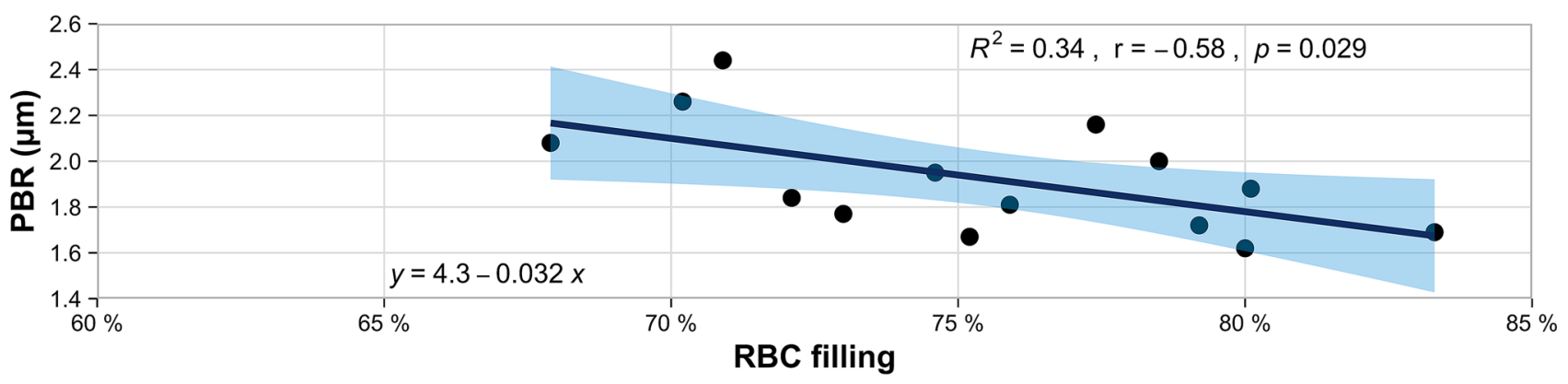

\section{b Post-exercise}

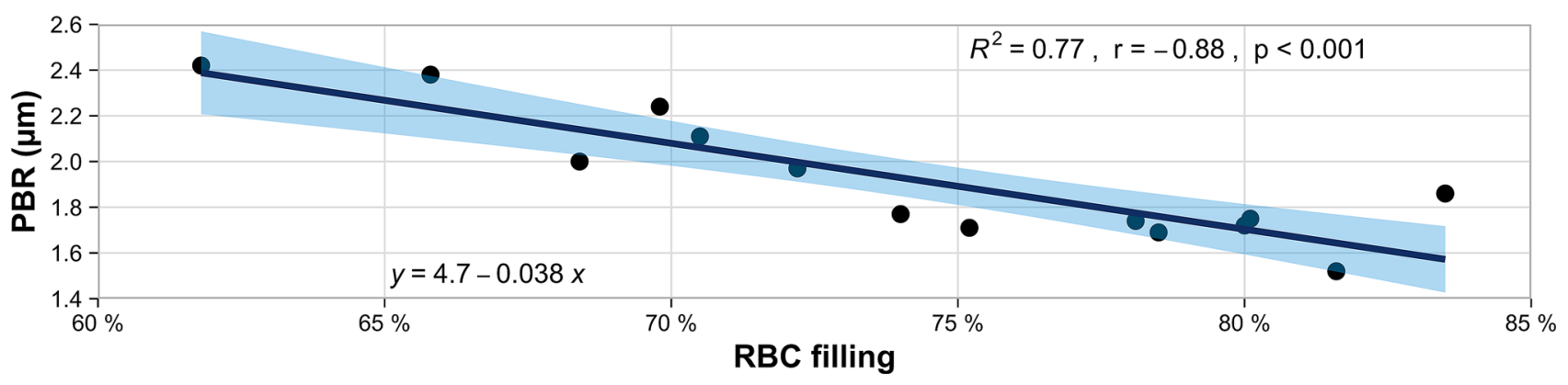

Fig. 2 a Scatterplot of PBR (\%) and RBC filling (\%) at baseline. b Scatterplot of PBR (\%) and RBC filling (\%) post-exercise. Linear regression lines overlaid, coefficients of determination, Spearman's correlation coefficients and p-values are given

percentage was maintained and even more pronounced after aerobic exercise, resulting also in a distinct association between change of PBR and change of RBC filling percentage. This aerobic exercise-stimulated association between the decrease of PBR and an increase of RBC filling percentage suggests that the endothelium and its ESL facilitate beneficial conditions for efficient perfusion in a hyperdynamic state like aerobic exercise.

In a previously published study, healthy volunteers performed an all-out running high-intensity interval training with one weekly session for four weeks [19]. Maximal exercise performance capacity (as measured by an incremental continuous running test) and PBR showed a negative correlation at baseline. The change in exercise performance capacity and the change in PBR showed an intensified negative correlation after the intervention indicating greater ESL thickness with improved exercise performance capacity [19]. It is intriguing to speculate that repeated endurance or highintensity interval training might have a protective effect on the microvasculature as we did not find an immediate effect 
Fig. 3 Scatterplot of change of PBR (\%) and change of RBC filling $(\%)$. Linear regression lines overlaid, coefficients of determination, Spearman's correlation coefficients and $\mathrm{p}$-values are given

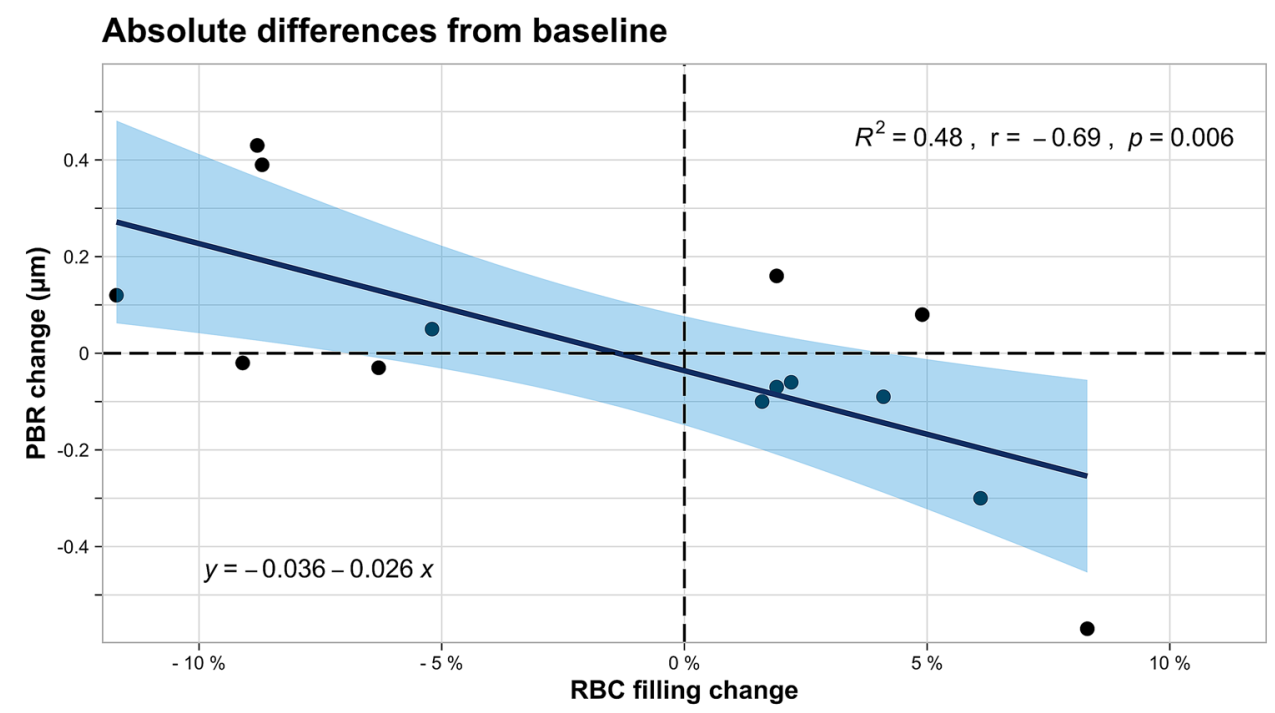

of a single aerobic-exercise on ESL dimension in our study. The underlying mechanisms have yet to be elucidated. A possible mechanism being discussed is an enhanced resistance of the ESL following repeated training sessions. For instance, moderate-intensity endurance training four times a week for 20 weeks performed by untrained young men lead to reduced syndecan- 1 and heparan sulphate levels at rest and lower levels of these ESL degradation parameters after an incremental cycling test until exhaustion following the intervention [20]. Interestingly, maximal incremental cycling until exhaustion in 21 untrained men (mean age: 23 years) did not affect syndecan-1 and heparan sulphate levels [21] as opposed to an acute bicycle test with $45 \mathrm{~min}$ at an intensity of $70 \%$ of individual maximum oxygen uptake performed in individuals with a mean age of 50 yrs that led to an increase in levels of syndecan-1 and syndecan-4 [22]. Aging appears to be a factor contributing to ESL integrity. Lee et al. conclude that increased blood flow causes ESL degradation [22]. As we did not measure ESL shedding parameters, we do not know whether the preserved ESL dimensions were accompanied by stable levels of ESL shedding parameters or not. In addition, we did not assess maximum performance capacity before and after an intervention but were interested in the effect of a onetime aerobic-exercise on PBR. Hence, we cannot offer data regarding the association of individual performance capacity and PBR. Running time and PBR did not show a significant relation (data not shown). Neither valid nor total capillary density, nor the ratio of valid to total capillary density did change post-exercise. Hence, immediate capillary recruitment after a single aerobic-exercise was not detectable in our study but might be present when exercise is performed regularly or when measured in athletes. Patients with chronic heart failure have a lower valid and total capillary density, but a higher ratio of valid to total capillary density [23]. It suggests perfused capillary recruitment in an otherwise rarefied microcirculation. This increased ratio is also seen with age, body-mass-index, total cholesterol, and Framingham risk score [24] and may indicate a chronic adaptation process.

This was the first field study to assess the effect of aerobic exercise on ESL dimensions. ESL reactions of the sublingual mucosa may differ from tissues with high oxygen demand as heart and skeletal muscle in such scenarios. Participants older of age, concomitant arterial hypertension, dyslipidaemia or dysglycaemia may show other endothelial reactivity and tissue perfusion compared to the healthy volunteers we studied. As we did not conduct invasive measurements, we were not able to assess lactate levels.

We report the feasibility of an alternative scenario for intravital microscopy with automised analyses other than an inhospital or a laboratory setting. We conclude that the endothelium of the microvasculature facilitates efficient perfusion in vessels reacting with an increased endothelial surface layer.

Author contributions Conceptualisation: TA; Funding acquisition, methodology, project administration and resources: TA; Investigation: AF, TN, HD, AH, TA; Software: HV; Formal analysis: AF, TN, HV; Visualisation: TN; Writing —original draft preparation: AF, TA; Writing-review and editing: AF, TN, HD, AH, HV, TA.

Funding Open Access funding enabled and organized by Projekt DEAL. This work was supported by intramural funding of the research group 'Klinische Forschung und Lehre' to T.A., University Hospital of Cologne, Department of Anaesthesiology and Intensive Care Medicine, Cologne, Germany.

\section{Compliance with ethical standards}

Conflict of interest Hans Vink is co-founder and chief science officer of Microvascular Health Solutions. The other authors report no conflict of interest. 
Ethical approval All procedures performed in studies involving human participants were in accordance with the ethical standards of the institutional research committee (Ethics Committee of the University of Cologne, reference number AZ 18-162) and with the 1964 Helsinki declaration and its later amendments.

Consent to participate All participants gave written informed consent prior to any study-related data acquisition.

Consent for publication The article is not under consideration for publication elsewhere. Publication of the article is approved by all authors and tacitly or explicitly by the responsible authorities where the work was carried out. All authors take responsibility for all aspects of the reliability and freedom from bias of the data presented and their discussed interpretation.

Open Access This article is licensed under a Creative Commons Attribution 4.0 International License, which permits use, sharing, adaptation, distribution and reproduction in any medium or format, as long as you give appropriate credit to the original author(s) and the source, provide a link to the Creative Commons licence, and indicate if changes were made. The images or other third party material in this article are included in the article's Creative Commons licence, unless indicated otherwise in a credit line to the material. If material is not included in the article's Creative Commons licence and your intended use is not permitted by statutory regulation or exceeds the permitted use, you will need to obtain permission directly from the copyright holder. To view a copy of this licence, visit http://creativecommons.org/licenses/by/4.0/.

\section{References}

1. Reitsma S, Slaaf DW, Vink H, van Zandvoort MAMJ, oude Egbrink MGA. The endothelial glycocalyx: composition, functions, and visualization. Pflügers Archiv. 2007;454(3):345-59. https://doi.org/10.1007/s00424-007-0212-8.

2. Becker BF, Chappell D, Bruegger D, Annecke T, Jacob M. Therapeutic strategies targeting the endothelial glycocalyx: acute deficits, but great potential. Cardiovasc Res. 2010;87(2):300-10. https ://doi.org/10.1093/cvr/cvq137.

3. Annecke T, Fischer J, Hartmann H, Tschoep J, Rehm M, et al. Shedding of the coronary endothelial glycocalyx: effects of hypoxia/reoxygenation vs ischaemia/reperfusion. Br J Anaesth. 2011;107(5):679-86. https://doi.org/10.1093/bja/aer269.

4. Becker BF, Fischer J, Hartmann H, Chen CC, Sommerhoff CP, Tschoep J, Conzen PC, Annecke T. Inosine, not adenosine, initiates endothelial glycocalyx degradation in cardiac ischemia and hypoxia. Nucleosides Nucleotides Nucleic Acids. 2011;30(12):1161-7.

5. Bogner-Flatz V, Braunstein M, Ocker LE, Kusmenkov T, Tschoep J, et al. On-the-scene hyaluronan and syndecan-1 serum concentrations and outcome after cardiac arrest and resuscitation. Mediators Inflamm. 2019;2019:8071619. https://doi. org/10.1155/2019/8071619.

6. Oshima K, Haeger SM, Hippensteel JA, Herson PS, Schmidt EP. More than a biomarker: the systemic consequences of heparan sulfate fragments released during endothelial surface layer degradation (2017 Grover Conference Series). Pulm Circ. 2018;8(1):2045893217745786. https://doi.org/10.1177/20458 93217745786.

7. Johansson PI, Stensballe J, Rasmussen LS, Ostrowski SR. A high admission syndecan-1 level, a marker of endothelial glycocalyx degradation, is associated with inflammation, protein $\mathrm{C}$ depletion, fibrinolysis, and increased mortality in trauma patients. Ann Surg.
2011;254(2):194-200. https://doi.org/10.1097/SLA.0b013e3182 26113d.

8. Hohn A, Baumann A, Pietroschinsky E, Franklin J, Illerhaus A, et al. Haemoadsorption: effective in reducing circulating fragments of the endothelial glycocalyx during cardiopulmonary bypass in patients undergoing on-pump cardiac surgery? Minerva Anestesiol. 2020. https://doi.org/10.23736/S0375-9393.20.14525 -5 .

9. Fuchs A, Groß S, Neumann T, Illerhaus A, Vink H, et al. Immediate effects of whole blood donation on the endothelial surface layer and glycocalyx shedding. Blood Transfus. 2020. https://doi. org/10.2450/2020.0025-20.

10. Jaarsma C, Vink H, van Haare J, Bekkers SCAM, van Rooijen BD, et al. Non-invasive assessment of microvascular dysfunction in patients with microvascular angina. Int J Cardiol. 2017;248:4339. https://doi.org/10.1016/j.ijcard.2017.05.010.

11. Koning NJ, Vonk ABA, Vink H, Boer C. Side-by-side alterations in glycocalyx thickness and perfused microvascular density during acute microcirculatory alterations in cardiac surgery. Microcirculation. 2016;23(1):69-74. https://doi.org/10.1111/micc.12260.

12. Dane MJC, Khairoun M, Lee DH, Van Den Berg BM, Eskens $\mathrm{BJM}$, et al. Association of kidney function with changes in the endothelial surface layer. Clin J Am Soc Nephrol. 2014;9(4):698704. https://doi.org/10.2215/cjn.08160813.

13. Martens RJH, Vink H, van Oostenbrugge RJ, Staals J. Sublingual microvascular glycocalyx dimensions in lacunar stroke patients. Cerebrovasc Dis. 2013;35(5):451-4. https://doi. org/10.1159/000348854.

14. Donati A, Damiani E, Domizi R, Romano R, Adrario E, et al. Alteration of the sublingual microvascular glycocalyx in critically ill patients. Microvasc Res. 2013;90:86-9. https://doi. org/10.1016/j.mvr.2013.08.007.

15. Lee DH, Dane MJC, van den Berg BM, Boels MGS, van Teeffelen JW, et al. Deeper penetration of erythrocytes into the endothelial glycocalyx is associated with impaired microvascular perfusion. PLoS ONE. 2014;9(5):e96477. https://doi.org/10.1371/journ al.pone.0096477.

16. Wadowski PP, Steinlechner B, Zimpfer D, Schlöglhofer T, Schima $\mathrm{H}$, et al. Functional capillary impairment in patients with ventricular assist devices. Sci Rep. 2019;9(1):5909. https://doi. org/10.1038/s41598-019-42334-3.

17. R Core Team. R: a language and environment for statistical computing. Vienna: R Foundation for Statistical Computing; 2019.

18. Wickham H. ggplot2: elegant graphics for data analysis. New York: Springer; 2016.

19. Schmitz B, Niehues H, Lenders M, Thorwesten L, Klose A, et al. Effects of high-intensity interval training on microvascular glycocalyx and associated microRNAs. Am J Physiol Heart Circ Physiol. 2019;316(6):H1538-51. https://doi.org/10.1152/ajphe art.00751.2018.

20. Majerczak J, Grandys M, Duda K, Zakrzewska A, Balcerczyk A, et al. Moderate-intensity endurance training improves endothelial glycocalyx layer integrity in healthy young men. Exp Physiol. 2017;102(1):70-85. https://doi.org/10.1113/ep085887.

21. Majerczak J, Duda K, Chlopicki S, Bartosz G, Zakrzewska A, et al. Endothelial glycocalyx integrity is preserved in young, healthy men during a single bout of strenuous physical exercise. Physiol Res. 2016;65:281-91.

22. Lee S, Kolset SO, Birkeland KI, Drevon CA, Reine TM. Acute exercise increases syndecan- 1 and -4 serum concentrations. Glycoconj J. 2019;36(2):113-25. https://doi.org/10.1007/s10719-01909869-z.

23. Wadowski PP, Hülsmann M, Schörgenhofer C, Lang IM, Wurm $\mathrm{R}$, et al. Sublingual functional capillary rarefaction in chronic heart failure. Eur J Clin Invest. 2018;48(2):e12869. https://doi. org/10.1111/eci.12869. 
24. Gu Y-M, Wang S, Zhang L, Liu Y-P, Thijs L, et al. Characteristics and determinants of the sublingual microcirculation in populations of different ethnicity. Hypertension. 2015;65(5):993-1001. https ://doi.org/10.1161/HYPERTENSIONAHA.114.05119.
Publisher's Note Springer Nature remains neutral with regard to jurisdictional claims in published maps and institutional affiliations. 